\title{
Less Conservative Control Design for Linear Systems with Polytopic Uncertainties via State-Derivative Feedback
}

\author{
Emerson R. P. da Silva, Edvaldo Assunção, \\ Marcelo C. M. Teixeira, and Luiz Francisco S. Buzachero \\ Research Laboratory in Control, Department of Electrical Engineering, Univ Estadual Paulista (UNESP), \\ Campus de Ilha Solteira, Avenue José Carlos Rossi, no 1370, 15385-000 Ilha Solteira, SP, Brazil
}

Correspondence should be addressed to Emerson R. P. da Silva, e.ravazzi@bol.com.br

Received 4 August 2011; Accepted 20 October 2011

Academic Editor: F. Lobo Pereira

Copyright (C) 2012 Emerson R. P. da Silva et al. This is an open access article distributed under the Creative Commons Attribution License, which permits unrestricted use, distribution, and reproduction in any medium, provided the original work is properly cited.

\begin{abstract}
The motivation for the use of state-derivative feedback instead of conventional state feedback is due to its easy implementation in some mechanical applications, for example, in vibration control of mechanical systems, where accelerometers have been used to measure the system state. Using linear matrix inequalities (LMIs) and a parameter-dependent Lyapunov functions (PDLF) allowed by Finsler's lemma, a less conservative approach to the controller design via state-derivative feedback, is proposed in this work, with and without decay rate restriction, for continuoustime linear systems subject to polytopic uncertainties. Finally, numerical examples illustrate the efficiency of the proposed method.
\end{abstract}

\section{Introduction}

In studies of classical control theory, it is well known that the use of state-derivative feedback $\left(u(t)=-K_{d} \dot{x}(t)\right)$ can be very useful, and in some cases, essential and advantageous for achieving desired performance in dynamic systems [1]. The interest to study the statederivative feedback comes from the fact that in systems using accelerometers, it is easier to get the state-derivatives signals than the states signals. Using the acceleration signal, the velocity can be obtained with good accuracy. However, it is more difficult to obtain the displacement [2]. Thus, the signals used in feedback are: velocity and acceleration. Respectively, these are the derivatives of position and velocity that can represent the system states. Due to its simple structure and low operating cost, accelerometers have been used in industry for solving various engineering problems. For example, they can give some applications: in vibration control of suspension bridges cables [3], in vibration control of components for aircraft 
landing [4], in active suspension systems of vehicles [5, 6], and in control of mechanical systems oscillations [7].

The use of state-derivative feedback in linear systems has been well explored in recent years. Some studies try to develop similar techniques for the existing state feedback. For example, [2] developed a generalized Ackermann formula for linear systems (SISO) with state-derivative feedback, [8] presented the design of a linear quadratic regulator (LQR), [9] proved that linear systems whose output is the state derivative are not observable nor stabilizable via output feedback, and [10] presented a way to get the state-derivative controller from the usual state feedback. A comparative study involving the state feedback and state-derivative feedback in linear time invariant systems is presented in [11]. An approach to the stabilizability and stability robustness of state-derivative feedback, including the fragility, can be seen in [12]. Other papers that include the design of controllers for mechanical systems and systems of vibration absorbers using state-derivative feedback can be found in [13-17].

In literature, there can be found books and papers that include the use of statederivative feedback in linear systems, with or without uncertainties in the plant, using techniques based on linear matrix inequalities (LMI) [18]. Projects using LMIs have the advantage of easily allowing the inclusion of uncertainties, for example, of polytopic type, and performance indices in the problem approach. Moreover, LMIs can be solved efficiently on microcomputers, using, for example, the MatLab software [19]. The controllers design, for linear or uncertain linear systems, is the most common concept comprising this study; see references: [20-24]. In [25], a LMI formulation is presented for controllers design that guarantees stability with and without decay rate of linear or uncertain linear systems. This method uses a common quadratic Lyapunov function (CQLF) to ensure system stability. The quadratic stability has proved to be very efficient, having solved many problems by convex optimization. However, recent literature results proved that the CQLFs generate some conservatism in the context of uncertain systems; that is, some problems may have no solution with CQLFs [26]. Thus, some studies have been developed using other types of Lyapunov functions in order to assure less conservative stability conditions. Among the results, we can name, for example, the parameter-dependent Lyapunov functions (PDLF) [27-30].

In order to obtain less conservative results than the conditions presented in [25], in [31] the authors presented LMI-based formulations in PDLFs for the design of state-derivative feedback applied to uncertain linear systems. The projects aim at the stability of systems with and without restriction in the decay rate. At first glance, it seems to be easier and less conservative to guarantee stability conditions using PDLFs instead of CQLFs. However, this was possible only for designs considering the decay rate. For designs considering only the system stability, the LMIs based on CQLFs [25] still had a slight advantage over the method presented in [31].

In order to obtain less conservative results than those presented in [25] and in [31], LMI-based techniques are proposed in this work, with and without restriction of the decay rate in state-derivative feedback for uncertain linear systems or subject to structural failure. Applying Finsler's Lemma [32] on parameter-dependent Lyapunov functions, less conservative robust conditions are obtained for the stability of dynamical systems. It is now seen that, considering only the stabilization of the system or stabilization with decay rate, the proposed method has significant advantages over the techniques mentioned above.

The efficiency of the proposed methodology is illustrated through numerical examples. 
Notation. The following notations are used in the text: $\left({ }^{T}\right)$ indicates transposition of a vector or matrix; $\left({ }^{-T}\right)$ indicates the inverse of the transposed matrix; $(*)$ indicates transposed blocks in a symmetric matrix; $\operatorname{diag}(\cdot, \cdot, \ldots, \cdot)$ denotes a block diagonal matrix.

\section{State-Derivative Feedback Control in Linear Systems with Polytopic Uncertainties}

Consider a linear system with time-invariant uncertainties, described as the state space variables as in $(2.1)$

$$
\dot{x}(t)=A(\xi) x(t)+B(\xi) u(t), \quad x\left(t_{0}\right)=x_{0},
$$

where $A(\xi) \in \mathbb{R}^{n \times n}$ and $B(\xi) \in \mathbb{R}^{n \times m}$ are matrices that represent the dynamics of the uncertain system, $x(t) \in \mathbb{R}^{n}$ is the state vector, and $u(t) \in \mathbb{R}^{m}$ is the control input vector.

The $(A, B)(\xi)$ matrices are represented by the convex combination of vertices, described by [18]

$$
(A, B)(\xi)=\left\{\sum_{i=1}^{r} \xi_{i}(A, B)_{i}, \quad \xi_{i} \in \mathcal{C}\right\},
$$

where $r$ is given by $r=2^{\phi}$, and $\phi$ is the number of uncertain components of the $(A, B)(\xi)$ matrices, and $(A, B)_{i}$ represent the vertices of the polytope. The $\xi_{i}$ parameters are unknown real constants, belonging to a $\operatorname{simplex} \operatorname{set} \mathcal{C}$, that is, satisfying the relation:

$$
\mathcal{C}=\left\{\begin{array}{c}
\xi_{i} \geq 0, \quad i=1,2, \ldots, r, \\
\sum_{i=1}^{r} \xi_{i}=1, \quad \xi=\left[\begin{array}{llll}
\xi_{1} & \xi_{2} & \ldots & \xi_{r}
\end{array}\right]^{T}
\end{array}\right\}
$$

Assuming that the system (2.1) has no null eigenvalues $(\operatorname{det}(A(\xi)) \neq 0)[2]$, the objective is to find a constant matrix $K_{d} \in \mathbb{R}^{m \times n}$, such that, when feedback into the system (2.1) with the control input

$$
u(t)=-K_{d} \dot{x}(t),
$$

the closed-loop system given by (2.1) and (2.4) is asymptotically stable and the matrix $(I+$ $\left.B(\xi) K_{d}\right)$ is invertible $\left(\operatorname{det}\left(I+B(\xi) K_{d}\right) \neq 0\right)$. Thus, the closed-loop system can be represented by

$$
\dot{x}(t)=A(\xi) x(t)-B(\xi) K_{d} \dot{x}(t) \Longleftrightarrow \dot{x}(t)=\left(I+B(\xi) K_{d}\right)^{-1} A(\xi) x(t),
$$

or by

$$
0=A(\xi) x(t)-\left(I+B(\xi) K_{d}\right) \dot{x}(t)
$$

which is equivalent to expression (2.5). 
The controller design is accomplished using the concept of asymptotic stability by analyzing the existence of a parametric Lyapunov function as in (2.7), for all $x(t) \neq 0$ [27]:

$$
\begin{gathered}
V(x(t), \xi)=x(t)^{T} P(\xi) x(t)>0, \quad P(\xi)=P(\xi)^{T} \in \mathbb{R}^{n \times n} \\
\dot{V}(x(t), \xi)=\dot{x}(t)^{T} P(\xi) x(t)+x(t)^{T} P(\xi) \dot{x}(t)<0
\end{gathered}
$$

where $P(\xi)$ is given by

$$
P(\xi)=\sum_{i=1}^{r} \xi_{i} P_{i}, \quad \xi_{i} \in \mathcal{C} .
$$

In the LMIs modelling, Lemma 2.1 is used.

Lemma 2.1 (Finsler's lemma). Consider $\mathcal{W} \in \mathbb{R}^{2 n}, \Phi(\xi) \in \mathbb{R}^{2 n \times 2 n}$ and $\boldsymbol{B}(\xi) \in \mathbb{R}^{n \times 2 n}$ with rank $(\boldsymbol{B}(\xi))<\delta$ and $\mathbb{B}(\xi)^{\perp}$ a basis for the null space of $\boldsymbol{B}(\xi)$ (in other words $\mathbb{B}(\xi) \mathcal{B}(\xi)^{\perp}=0$ ).

Then, the following conditions are equivalent:

(i) $\mathcal{W}^{T} \Phi(\xi) \mathcal{W}<0$, for all $\mathcal{W} \neq 0, \boldsymbol{B}(\xi) \mathcal{W}=0$,

(ii) $B(\xi)^{\perp^{T}} \Phi(\xi) B(\xi)^{\perp}<0$,

(iii) $\exists \rho \in \mathbb{R}: \Phi(\xi)-\rho \mathbb{B}(\xi)^{T} \mathbb{B}(\xi)<0$,

(iv) $\exists Q \in \mathbb{R}^{2 n \times n}: \Phi(\xi)+Q \mathcal{B}(\xi)+B(\xi)^{T} Q^{T}<0$,

where $\rho$ and $Q$ are called extra variables (or multipliers).

Proof. See $[27,32]$.

Lemma 2.1 can be used to study a set of matrix inequalities without an explicit multiplication between the matrices of the system (2.6) with the matrices $P(\xi)$ of the Lyapunov function. This Lemma is widely used in many LMI-based control applications, so as to eliminate variables, decouple matrices, or reduce the number of LMIs in control design $[33,34]$.

At first, we define the following vectors and matrices:

$$
\begin{gathered}
\mathcal{W}=\left[\begin{array}{c}
x(t) \\
\dot{x}(t)
\end{array}\right], \quad \mathscr{D}(P(\xi))=\left[\begin{array}{cc}
0 & P(\xi) \\
P(\xi) & 0
\end{array}\right], \\
\mathcal{Q}=\left[\begin{array}{l}
X \\
X
\end{array}\right], \quad B(\xi)=\left[A(\xi)-I-B(\xi) K_{d}\right],
\end{gathered}
$$

where $\mathrm{X}$ is any nonsingular matrix of appropriate dimensions.

Using these definitions, the closed-loop system (2.6) and the stability condition (2.7) can be rewritten respectively as

$$
\begin{gathered}
B(\xi) \mathcal{W}=0, \\
\dot{V}(\mathcal{W}, \xi)=\mathcal{W}^{T} \boldsymbol{\Phi}(P(\xi)) \mathcal{W}<0, \quad \forall \mathcal{W} \neq 0 .
\end{gathered}
$$

During the demonstration of the theorems proposed below, Lemma 2.2 is also used. 
Lemma 2.2. For every nonsymmetric matrix $M\left(M \neq M^{T}\right)$, if $M+M^{T}<0$, then $M$ is invertible.

Proof. See [18].

With this information, in Theorem 2.3, sufficient conditions for the stability of system (2.6) are proposed.

Theorem 2.3. If there exist symmetric matrices $Q_{i} \in \mathbb{R}^{n \times n}$, matrices $G \in \mathbb{R}^{m \times n}$, and $Y \in \mathbb{R}^{n \times n}$, satisfying the LMI

$$
\left[\begin{array}{cc}
A_{i} Y^{T}+Y A_{i}^{T} & Q_{i}-Y^{T}-B_{i} G+Y A_{i}^{T} \\
* & -Y^{T}-Y-B_{i} G-G^{T} B_{i}^{T}
\end{array}\right]<0,
$$

where $i=1,2, \ldots, r$, then, the system (2.6) is stabilizable, and a controller that solves the problem can be found as in

$$
K_{d}=G Y^{-T}
$$

Proof. Suppose (2.11) is feasible. Replacing $Q_{i}=X^{-1} P_{i} X^{-T}, G=K_{d} X^{-T}$, and $Y=X^{-1}$ in (2.11), multiplying each element by $\xi_{i}$ and summing for all $i=1,2, \ldots, r,(2.13)$ follows

$$
\left[\begin{array}{cc}
\sum_{i=1}^{r} \xi_{i} A_{i} X^{-T}+X^{-1} \sum_{i=1}^{r} \xi_{i} A_{i}^{T} & X^{-1} \sum_{i=1}^{r} \xi_{i} P_{i} X^{-T}-X^{-T}-\sum_{i=1}^{r} \xi_{i} B_{i} K_{d} X^{-T}+X^{-1} \sum_{i=1}^{r} \xi_{i} A_{i}^{T} \\
* & -X^{-T}-X^{-1}-\sum_{i=1}^{r} \xi_{i} B_{i} K_{d} X^{-T}-X^{-1} K_{d}^{T} \sum_{i=1}^{r} \xi_{i} B_{i}^{T}
\end{array}\right]<0 .
$$

From (2.2) and (2.8), (2.14) follows

$$
\left[\begin{array}{cc}
A(\xi) X^{-T}+X^{-1} A(\xi)^{T} & X^{-1} P(\xi) X^{-T}-X^{-T}-B(\xi) K_{d} X^{-T}+X^{-1} A(\xi)^{T} \\
* & -X^{-T}-X^{-1}-B(\xi) K_{d} X^{-T}-X^{-1} K_{d}^{T} B(\xi)^{T}
\end{array}\right]<0 .
$$

Left-multiplying (2.14) by $\operatorname{diag}(X, X)$ and right-multiplying by $\operatorname{diag}\left(X^{T}, X^{T}\right),(2.15)$ follows

$$
\left[\begin{array}{cc}
X A(\xi)+A(\xi)^{T} X^{T} & P(\xi)-X+A(\xi)^{T} X^{T}-X B(\xi) K_{d} \\
* & -X-X^{T}-X B(\xi) K_{d}-K_{d}^{T} B(\xi)^{T} X^{T}
\end{array}\right]<0 .
$$

Multiplying (2.15) by (-1) and left- and right-multiplying by $\left[\begin{array}{ll}0 & I \\ I & 0\end{array}\right],(2.16)$ follows

$$
\left[\begin{array}{cc}
X+X B(\xi) K_{d}+X^{T}+K_{d}^{T} B(\xi)^{T} X^{T} & -P(\xi)+X^{T}-X A(\xi)+K_{d}^{T} B(\xi)^{T} X^{T} \\
* & -X A(\xi)-A(\xi)^{T} X^{T}
\end{array}\right]>0,
$$

so: $X+X B(\xi) K_{d}+X^{T}+K_{d}^{T} B(\xi)^{T} X^{T}>0 \Leftrightarrow X\left(I+B(\xi) K_{d}\right)+\left(I+K_{d}^{T} B(\xi)^{T}\right) X^{T}>0$. According to Lemma 2.2 , it follows that $X\left(I+B(\xi) K_{d}\right)$ is full rank, so $\left(I+B(\xi) K_{d}\right)$ and $X^{T}$ are full rank, that is, invertible. 
Note that (2.15) can be rewritten as

$$
\left[\begin{array}{cc}
0 & \mathrm{P}(\xi) \\
P(\xi) & 0
\end{array}\right]+\left[\begin{array}{cc}
X A(\xi)+A(\xi)^{T} X^{T} & -X+A(\xi)^{T} X^{T}-X B(\xi) K_{d} \\
* & -X-X B(\xi) K_{d}-X^{T}-K_{d}^{T} B(\xi)^{T} X^{T}
\end{array}\right]<0
$$

or

$$
\left[\begin{array}{cc}
0 & P(\xi) \\
P(\xi) & 0
\end{array}\right]+\left[\begin{array}{cc}
X A(\xi) & -X-X B(\xi) K_{d} \\
X A(\xi) & -X-X B(\xi) K_{d}
\end{array}\right]+\left[\begin{array}{cc}
A(\xi)^{T} X^{T} & A(\xi)^{T} X^{T} \\
-X^{T}-K_{d}^{T} B(\xi)^{T} X^{T} & -X^{T}-K_{d}^{T} B(\xi)^{T} X^{T}
\end{array}\right]<0
$$

Now, putting $X$ and $X^{T}$ in evidence, (2.19) follows

$$
\left[\begin{array}{cc}
0 & P(\xi) \\
P(\xi) & 0
\end{array}\right]+\left[\begin{array}{l}
X \\
X
\end{array}\right]\left[A(\xi)-I-B(\xi) K_{d}\right]+\left[A(\xi)-I-B(\xi) K_{d}\right]^{T}\left[\begin{array}{l}
X \\
X
\end{array}\right]^{T}<0 .
$$

Considering (2.9) and Lemma 2.1, $\mathcal{W}^{T} \Phi(\xi) \mathcal{W}<0$ is equivalent to $\Phi(\xi)+Q \mathcal{B}(\xi)+B(\xi)^{T} Q^{T}<0$, because $B(\xi) W=0$. From this it follows that if the LMI (2.11) is feasible, then there exists a symmetric parameter-dependent matrix $P(\xi)>0$ satisfying the conditions of Lyapunov (2.7) for the system (2.6). Thus, system (2.6), considering the gain (2.12), is asymptotically stable.

The stability of the system is not always enough, because there are projects that have performance constraints. A very important performance index is the restriction of decay rate, which is responsible for the rapid response (transient period) of the system [18].

\subsection{Decay Rate in State-Derivative Feedback Control with Polytopic Uncertainties}

Consider a candidate Lyapunov function of the type $V(x(t), \xi)=x(t)^{T} P(\xi) x(t)>0$, with $\dot{V}(x(t), \xi)<0$ for all $x(t) \neq 0$. The decay rate $\gamma>0$ is obtained if the condition

$$
\begin{gathered}
\dot{V}(x(t), \xi) \leq-2 \gamma V(x(t), \xi) \\
\Longleftrightarrow \dot{x}(t)^{T} P(\xi) x(t)+x(t)^{T} P(\xi) \dot{x}(t) \leq-2 \gamma x(t)^{T} P(\xi) x(t)
\end{gathered}
$$

is satisfied for every trajectory $x(t)$ of the system (2.6), $t \geq 0$ [18]. Figure 1 shows the region $\gamma$, where $\lambda$ are the eigenvalues of (2.6).

Defining the following vectors and matrices

$$
\begin{aligned}
& \mathcal{W}=\left[\begin{array}{l}
x(t) \\
\dot{x}(t)
\end{array}\right], \quad \boldsymbol{\Phi}(P(\xi))=\left[\begin{array}{cc}
2 \gamma P(\xi) & P(\xi) \\
P(\xi) & 0
\end{array}\right], \\
& \mathcal{Q}=\left[\begin{array}{l}
X \\
X
\end{array}\right], \quad B(\xi)=\left[A(\xi)-I-B(\xi) K_{d}\right],
\end{aligned}
$$

the stability condition with decay rate restriction (2.20) can be rewritten as (2.10). 


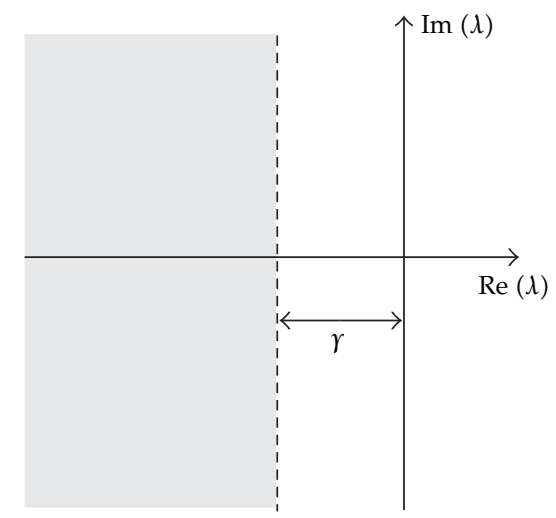

Figure 1: Region $\gamma$ for pole placement.

Knowing this, sufficient conditions for the stability of system (2.6) with decay rate $r>0$ are proposed in Theorem 2.4 .

Theorem 2.4. Given a real constant $\gamma>0$. If there exist symmetric matrices $Q_{i} \in \mathbb{R}^{n \times n}$, matrices $G \in \mathbb{R}^{m \times n}$, and $Y \in \mathbb{R}^{n \times n}$, satisfying the $L M I$

$$
\left[\begin{array}{cc}
2 \gamma Q_{i}+A_{i} Y^{T}+Y A_{i}^{T} & Q_{i}-Y^{T}-B_{i} G+Y A_{i}^{T} \\
* & -Y^{T}-Y-B_{i} G-G^{T} B_{i}^{T}
\end{array}\right]<0,
$$

where $i=1,2, \ldots, r$, then, the system (2.6) is stabilizable with decay rate $\gamma$, and a controller that solves the problem can be found by (2.12).

Proof. The proof follows similar steps from Theorem 2.3 proof, considering (2.21).

\section{Extended Results of Finsler's Lemma Applied to State-Derivative Feedback Control}

In the next theorems, a scalar $\mu>0$ is involved in the set of LMIs. It is possible to obtain less conservative results than the theorems presented above with an appropriate choice of $\mu$. The proposed Theorem 3.1 is an extension of Theorem 2.3.

Theorem 3.1. For a given arbitrary scalar $\mu>0$, if there exist symmetric matrices $Q_{i} \in \mathbb{R}^{n \times n}$, matrices $G \in \mathbb{R}^{m \times n}$, and $Y \in \mathbb{R}^{n \times n}$, satisfying the $L M I$

$$
\left[\begin{array}{cc}
A_{i} Y^{T}+Y A_{i}{ }^{T} & Q_{i}-Y^{T}-B_{i} G+\mu Y A_{i}{ }^{T} \\
* & -\mu Y^{T}-\mu Y-\mu B_{i} G-\mu G^{T} B_{i}{ }^{T}
\end{array}\right]<0,
$$

where $i=1,2, \ldots, r$, then, the system (2.6) is stabilizable, and a controller that solves the problem can be found by (2.12). 
Proof. The proof follows similar steps from Theorem 2.3 proof, considering

$$
Q=\left[\begin{array}{c}
X \\
\mu X
\end{array}\right]
$$

The proposed Theorem 3.2 is an extension of Theorem 2.4.

Theorem 3.2. Given a real constant $\gamma>0$ and an arbitrary scalar $\mu>0$, if there exist symmetric matrices $Q_{i} \in \mathbb{R}^{n \times n}$, matrices $G \in \mathbb{R}^{m \times n}$, and $Y \in \mathbb{R}^{n \times n}$, satisfying the LMI

$$
\left[\begin{array}{cc}
2 \gamma Q_{i}+A_{i} Y^{T}+Y A_{i}^{T} & Q_{i}-Y^{T}-B_{i} G+\mu Y A_{i}^{T} \\
* & -\mu Y^{T}-\mu Y-\mu B_{i} G-\mu G^{T} B_{i}^{T}
\end{array}\right]<0
$$

where $i=1,2, \ldots, r$, then, the system (2.6) is stabilizable with decay rate $\gamma$, and a controller that solves the problem can be found by (2.12).

Proof. The proof follows similar steps from Theorem 2.3 proof, considering (2.21) and (3.2).

Note 1. In Theorems 3.1 and 3.2 the scalar $\mu>0$ must be obtained through a one-dimensional search.

The tuning parameter $\mu$ was added in the LMIs (3.1) and (3.3) in an attempt to obtain less conservative stability conditions than Theorems 2.3 and 2.4, respectively. This procedure of adding scalar in LMIs has been widely explored in literature. For example, in [30, 31, $33,35]$, the authors can improve the LMI stability region by simply changing the parameter values.

The efficiency of the proposed methodology can be found in the solution of the examples discussed below.

\section{Examples-Digital Simulations}

Consider the vibration control system illustrated in Figure 2 [2].

The system's dynamics can be represented by the uncertain system (2.1), considering

$$
\begin{gathered}
A(\xi)=\left[\begin{array}{cccc}
0 & 0 & 1 & 0 \\
0 & 0 & 0 & 1 \\
\frac{-k_{1}-k_{2}}{m_{1}} & \frac{k_{2}}{m_{1}} & \frac{-b_{1}-b_{2}}{m_{1}} & \frac{b_{2}}{m_{1}} \\
\frac{k_{2}}{m_{2}} & \frac{-k_{2}}{m_{2}} & \frac{b_{2}}{m_{2}} & \frac{-b_{2}}{m_{2}}
\end{array}\right], \\
B(\xi)=\left[\begin{array}{llll}
0 & 0 & \frac{-1}{m_{1}} & \frac{1}{m_{2}}
\end{array}\right]^{T},
\end{gathered}
$$

with the state vector given by $x(t)=\left[\begin{array}{llll}x_{1}(t) & x_{2}(t) & \dot{x}_{1}(t) & \dot{x}_{2}(t)\end{array}\right]^{T}$, where, $x_{1}(t)$ and $x_{2}(t)$ are the vertical displacements of the masses $m_{1}$ and $m_{2}$, respectively, and $\dot{x}_{1}(t)$ and $\dot{x}_{2}(t)$ are their 


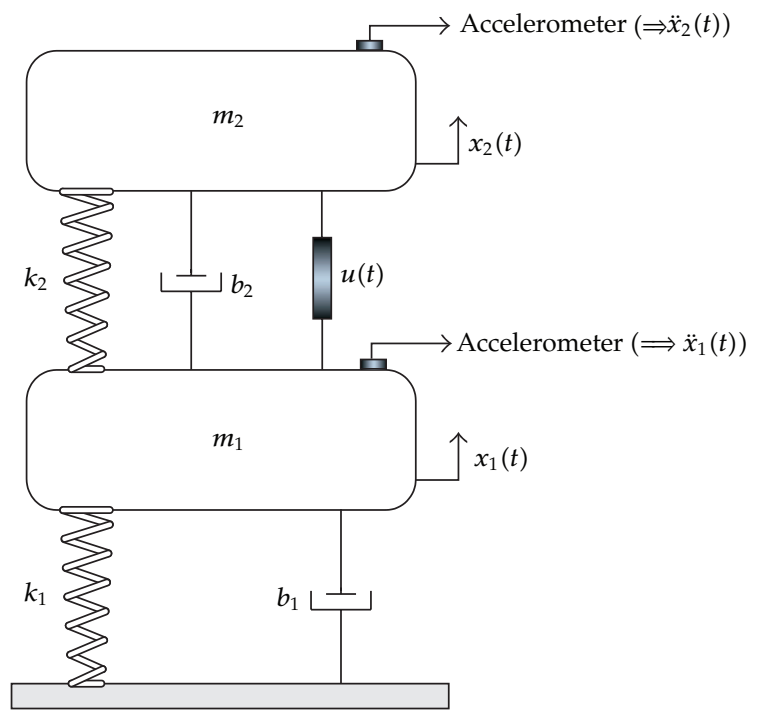

Figure 2: Mechanical system.

Table 1: Parameters of mechanical system.

\begin{tabular}{lccccc}
\hline$m_{1}$ & $m_{2}$ & $k_{1}$ & $k_{2}$ & $b_{1}$ & $b_{2}$ \\
\hline $100(\mathrm{~kg})$ & $10(\mathrm{~kg})$ & $360(\mathrm{kN} / \mathrm{m})$ & $36(\mathrm{kN} / \mathrm{m})$ & {$[0,70](\mathrm{Ns} / \mathrm{m})$} & $50(\mathrm{Ns} / \mathrm{m})$ \\
\hline
\end{tabular}

velocities. Parameters $k_{1}$ and $k_{2}$ are elastic constants and $b_{1}$ and $b_{2}$ damping constants. The respective values for the system parameters are shown in Table 1.

Assuming that the damping coefficient $b_{1}$ is uncertain and belongs to the interval $0 \leq$ $b_{1} \leq 70(\mathrm{Ns} / \mathrm{m})$ (i.e., the damper may break after some time of use, $\left.b_{1}=0\right)$. The objective of the control system is to reduce the vibration of the mass $m_{1}$ through of the controlled vibration of the mass $m_{2}$, with the control signal $u(t)$.

Using this information, the following vertices of the polytope are obtained:

$$
A_{1}=10^{3} \times\left[\begin{array}{cccc}
0 & 0 & 0.0010 & 0 \\
0 & 0 & 0 & 0.0010 \\
-3.9600 & 0.3600 & -0.0012 & 0.0005 \\
3.6000 & -3.6000 & 0.0050 & -0.0050
\end{array}\right]
$$

with $b_{1}=70 \mathrm{Ns} / \mathrm{m}$ in $A_{1}$,

$$
A_{2}=10^{3} \times\left[\begin{array}{cccc}
0 & 0 & 0.0010 & 0 \\
0 & 0 & 0 & 0.0010 \\
-3.9600 & 0.3600 & -0.0005 & 0.0005 \\
3.6000 & -3.6000 & 0.0050 & -0.0050
\end{array}\right] \text {, }
$$

with $b_{1}=0$ in $A_{2}$,

$$
B_{1}=B_{2}=\left[\begin{array}{llll}
0 & 0 & -0.01 & 0.10
\end{array}\right]^{T} .
$$


For the controllers design, MatLab software and the solver "LMILab" [19] were used. For this example, it was considered the following case.

\section{Stability}

Using the LMI (2.11) from Theorem 2.3, the controller in (4.5) was obtained using (2.12)

$$
K_{d}=10^{4} \times\left[\begin{array}{llll}
-4.3659 & 3.6076 & 0.0292 & 0.0075
\end{array}\right]
$$

The controlled system response to the initial condition of simulation $x(0)=$ $\left[\begin{array}{llll}0.05 & 0.05 & 0.2 & 0.2\end{array}\right]^{T}$ with damper $b_{1}$ working and after the failure $\left(b_{1}=0\right)$ can be seen in Figure 3. Referring to Figure 3, note that the behavior of the controlled system almost does not change, even after breaking the damper $b_{1}$. Thus, the designed controller was able to stabilize the system even after the occurrence of a structural failure.

Figure 4 shows the control signal effort considering controller (4.5) in control input (2.4).

Now, using the LMI (3.1) from Theorem 3.1 with $\mu=20$, the controller in (4.6) was obtained using (2.12)

$$
K_{d}=10^{4} \times\left[\begin{array}{llll}
-1.2108 & 1.0586 & 0.0041 & 0.0011
\end{array}\right]
$$

Figure 6 shows the control signal effort considering controller (4.6) in control input (2.4).

In this example, with a one-dimensional search in $\mu$, controller (4.6) found by Theorem 3.1 obtained a better performance considering the control signal input (Figure 6) than controller (4.5) found by Theorem 2.3 (Figure 4). However, there are no theoretical guarantees about the influence of $\mu$ in the performance of the closed-loop system. What we can guarantee is that there are systems that can be stabilized with Theorem 3.1 and cannot be with Theorem 2.3. This is verified later in Section 4.1.

Still looking at Figures 3 and 5, the duration of the transient period of the controlled system in both cases is around 3.5 seconds; that is, the tuning parameter $\mu$ has had limited influence in this regard. Whereas the system must have a lower transient, because in some cases this time may be too long, new projects can be done by adding the decay rate restriction. Using Theorems 2.4 and 3.2, a shorter transient period response can be found.

\section{Stability and Decay Rate}

Using the LMI (2.22) from Theorem 2.4 with $\gamma=0.99$ (maximum obtained for this example), the controller in (4.7) was obtained using (2.12)

$$
K_{d}=10^{3} \times\left[\begin{array}{llll}
-5.0893 & 4.5906 & 0.0577 & 0.0038
\end{array}\right] .
$$

The response of the controlled system with decay rate $\gamma=0.99$ with damper $b_{1}$ working and with damper $b_{1}$ broken (structural failure) can be seen in Figure 7. By examining Figure 7, it can be observed that the behavior of the controlled system is almost not affected, even after the occurrence of a failure in the damper $b_{1}$. Thus, the designed controller was able to secure the stability of the system and even reduce the settling time at around $1.5 \mathrm{~s}$. 


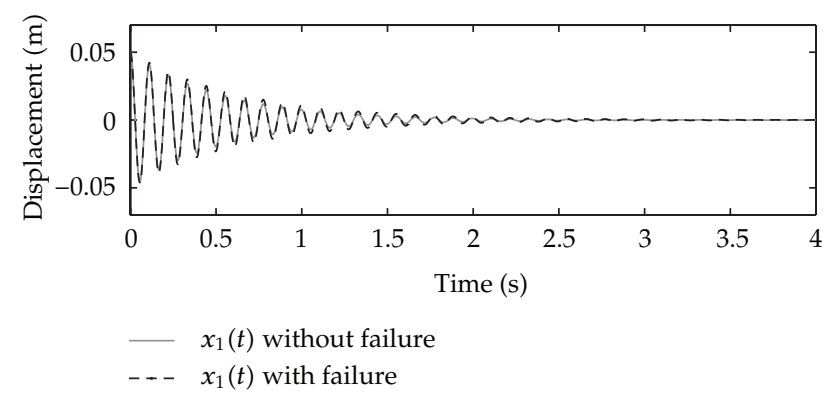

(a)

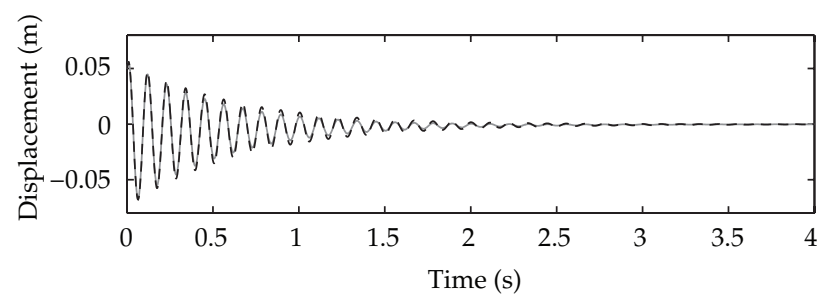

$x_{2}(t)$ without failure

$\ldots x_{2}(t)$ with failure

(b)

Figure 3: System's simulation (4.1) with controller (4.5).

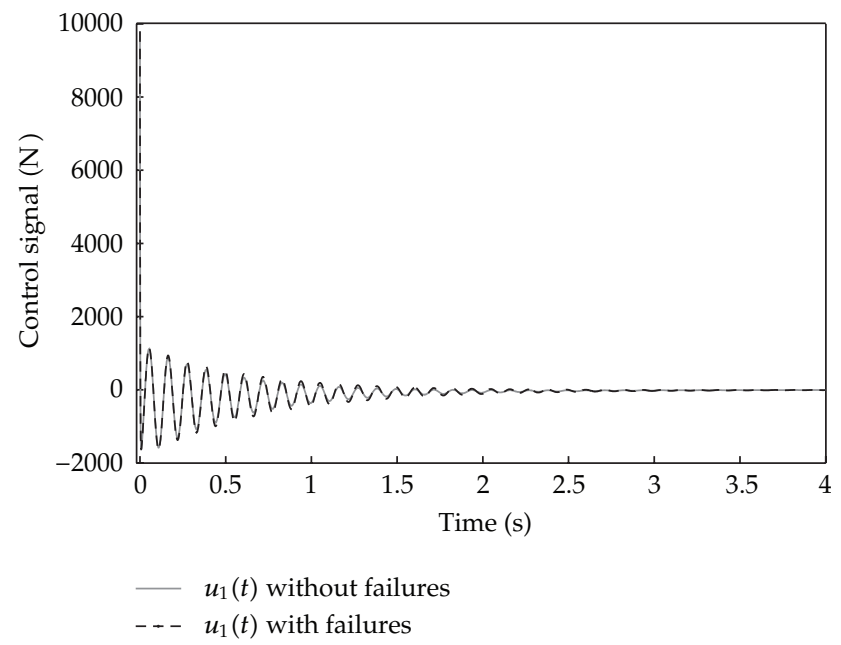

Figure 4: Control signal considering controller (4.5).

In Table 2, it is clear that the eigenvalues of the local models have real part lesser than $\gamma=0.99$. Therefore, the decay rate specification of the project has been met. There are four eigenvalues for each vertex.

Note 2. After lots of tests, through numerical examples, it was not possible to obtain values of $\gamma>0.99$ using the LMI (2.22) from Theorem 2.4. However, this is overcome by using Theorem 3.2. 


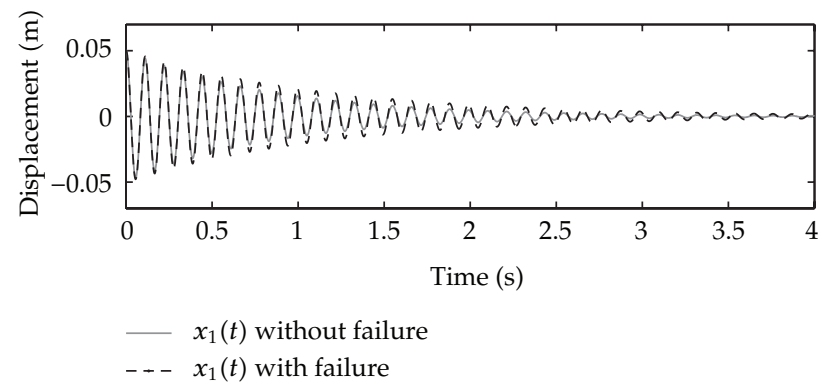

(a)

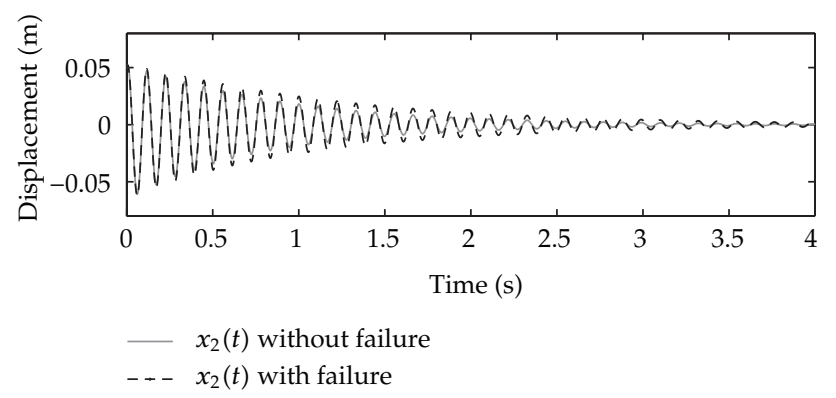

(b)

Figure 5: System's simulation (4.1) with controller (4.6).

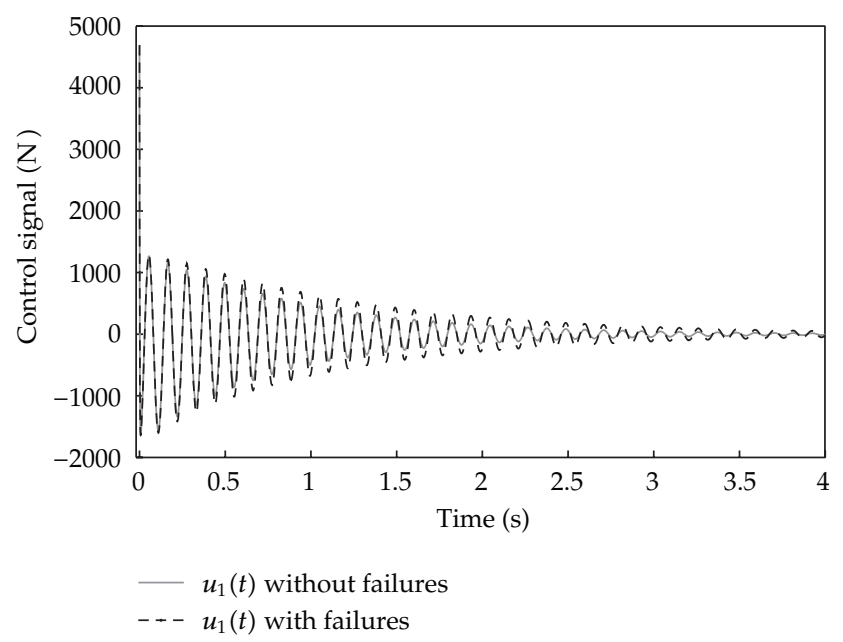

Figure 6: Control signal considering controller (2.6).

Figure 8 shows the control signal effort considering controller (4.7) in control input (2.4).

Using the LMI (3.3) from Theorem 3.2 with $\mu=0.01$ and $\gamma=10$, the controller in (4.8) was obtained using $(2.12)$

$$
K_{d}=10^{3} \times\left[\begin{array}{llll}
3.2028 & 1.0459 & 0.0573 & -0.0027
\end{array}\right] .
$$




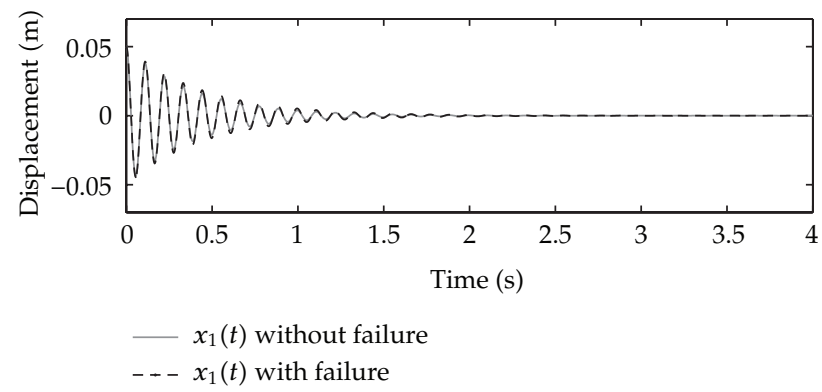

(a)

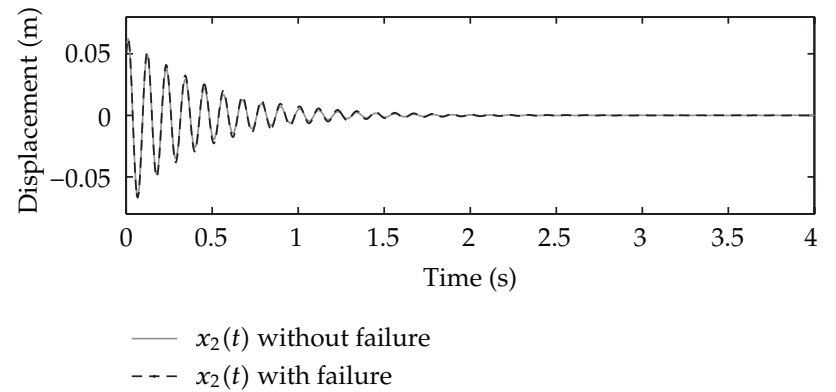

(b)

Figure 7: System's simulation (4.1) with controller (4.7).

Table 2: Eigenvalue position for system (4.1) with (4.7) state-derivative feedback gain.

\begin{tabular}{lc}
\hline Vertices & Eigenvalues \\
\hline \multirow{2}{*}{$A_{1}, B_{1}$} & $-2.5821 \pm \mathrm{j} 56.7358$ \\
& -629.6952 \\
\hline \multirow{2}{*}{$A_{2}, B_{2}$} & -7.9420 \\
& $-2.2683 \pm \mathrm{j} 56.7752$ \\
& -629.1198 \\
\hline
\end{tabular}

Note that with the addition of the tuning parameter $\mu$ in LMIs, it was possible to design controller with decay rate greater than $0.99(\gamma>0.99)$, which hitherto was not possible. Therefore, the use of the parameter $\mu$ on designs that consider the stabilization of the system with decay rate can establish influences in transient behavior of the controlled system, given that the greater the decay rate, the smaller the transient and, moreover, the control effort increases. Observing Figure 9, it can be seen that the controlled system, with or without fault, has settling time under $0.4 \mathrm{~s}$. Thus, the controller (4.8) was able to improve system performance comparing to responses of the controllers (4.5), (4.6), and (4.7). The response to (4.8) is approximately 12 times faster than the response with (4.5) and (4.6) and 7 times faster than (4.7).

In Table 3, it is clear that the eigenvalues of the local models have real part lesser than $r=10$. Therefore, the decay rate specification of the project has been met. There are four eigenvalues for each vertex. 


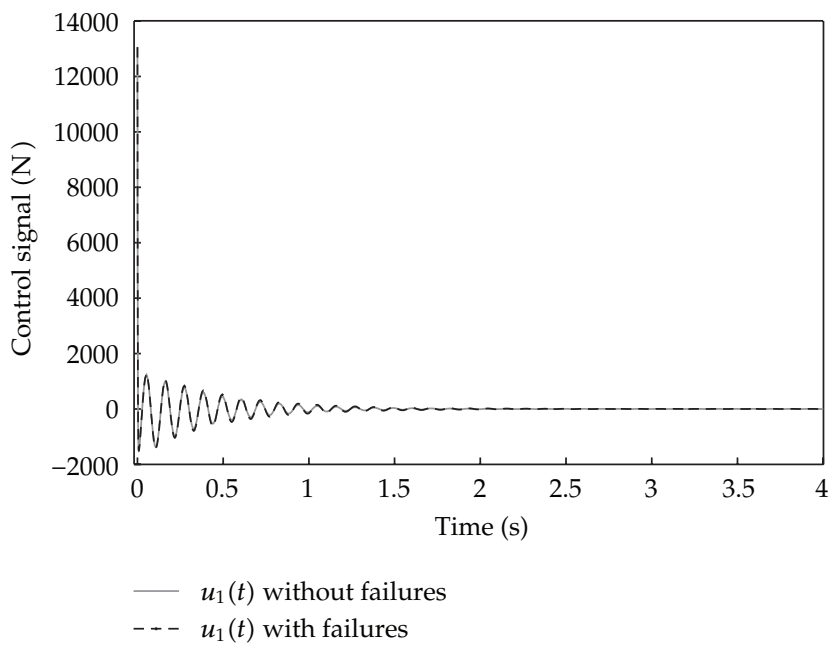

Figure 8: Control signal considering controller (4.7).

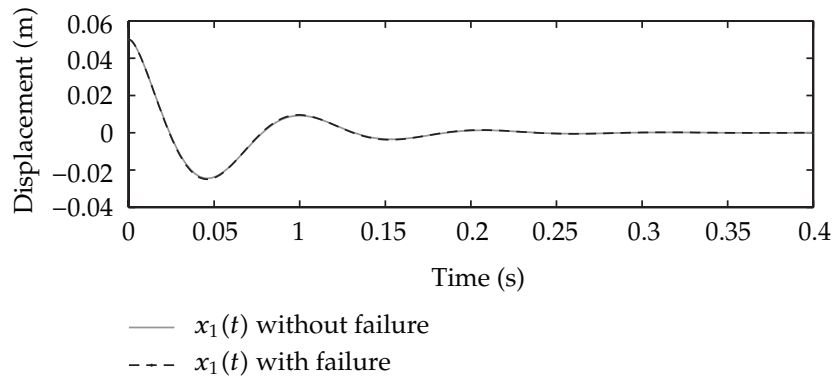

(a)

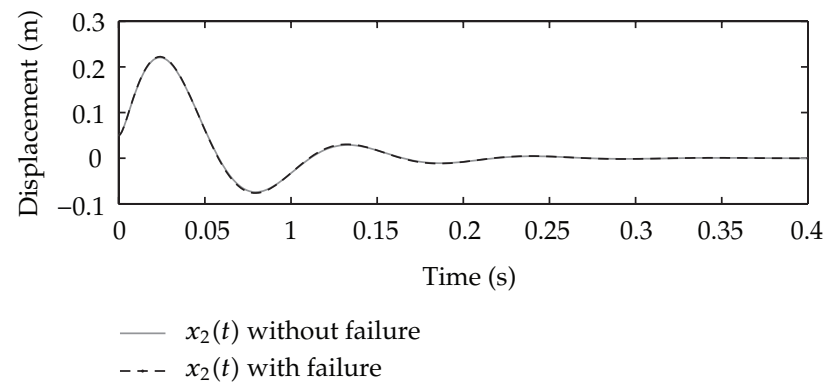

(b)

Figure 9: System's simulation (4.1) with controller (4.8).

Note 3. After lots of tests, the maximum value found for this example was $\gamma=162.88$ with $\mu=0.00001$. Considering these parameters, the controlled system has a settling time of approximately $0.04 \mathrm{~s}$. Consequently, there is a considerable increase of the control effort (approximately $\max |u(t)|=1 \times 10^{10} \mathrm{~N}$ ).

Figure 10 shows the control signal effort considering the controller (4.8) in the control input (2.4). 
Table 3: Eigenvalue position for system (4.1) with (4.8) state-derivative feedback controller.

\begin{tabular}{lc}
\hline Vertices & Eigenvalues \\
\hline \multirow{3}{*}{$A_{1}, B_{1}$} & $-17.9465 \pm \mathbf{j} 58.5021$ \\
& -403.4928 \\
& -53.8439 \\
$A_{2}, B_{2}$ & $-17.6585 \pm \mathbf{j} 58.8130$ \\
& -400.8770 \\
\hline
\end{tabular}

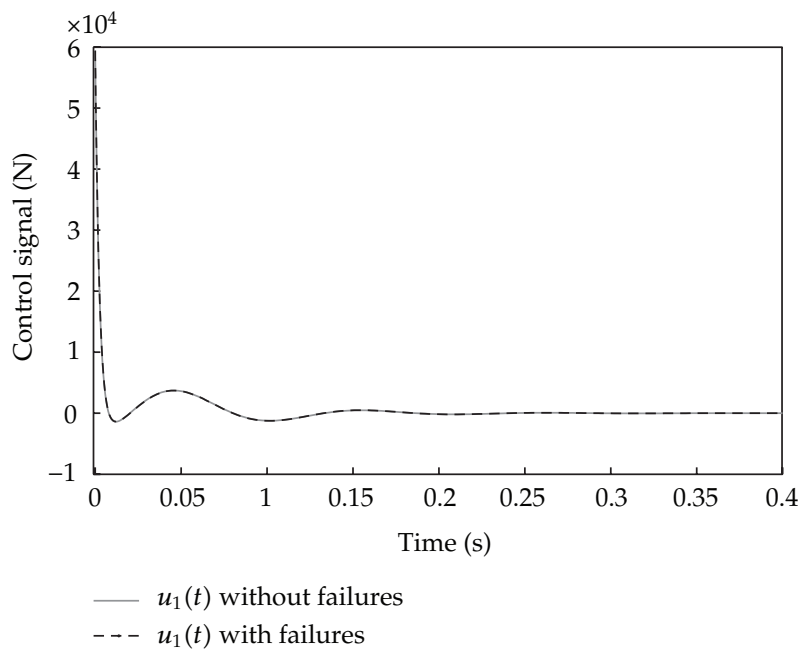

Figure 10: Control signal considering controller (4.8).

In the next example it is illustrated that the stability conditions considering $\mu$ in the design are less conservative.

\subsection{Example 2-Linear Systems with Polytopic Uncertainties}

Consider an uncertain linear system with the following polytope vertices:

$$
\begin{array}{ll}
A_{1}=\left[\begin{array}{cc}
20 & -100 \\
10 & 1
\end{array}\right], & B_{1}=\left[\begin{array}{l}
10 \\
10
\end{array}\right], \\
A_{2}=\left[\begin{array}{cc}
\zeta_{1} & -100 \\
10 & 30
\end{array}\right], & B_{2}=\left[\begin{array}{c}
\zeta_{2} \\
1
\end{array}\right] .
\end{array}
$$

\section{Stability}

The objective is to verify the feasibility points of Theorems 2.3 and 3.1 considering $-5 \leq \zeta_{1} \leq 5$ and $10 \leq \zeta_{2} \leq 15$. The feasibility region is shown in Figure 11 .

Considering the same uncertain linear system, Figure 12 shows the totality of controllers that could be found for various values of $\mu$, comprising $\mu \in[0.05,2.5]$. Analysing Figure 12, it can be observed that for small values of $\mu$ the totality of feasible designs is greater. 


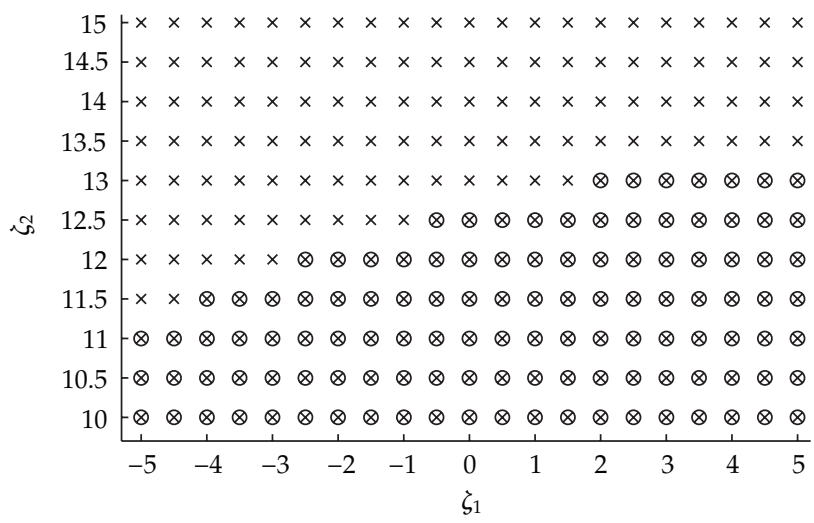

Figure 11: Controller design with Theorems $2.3(\circ)$ and $3.1(\mu=0.55, \times)$.

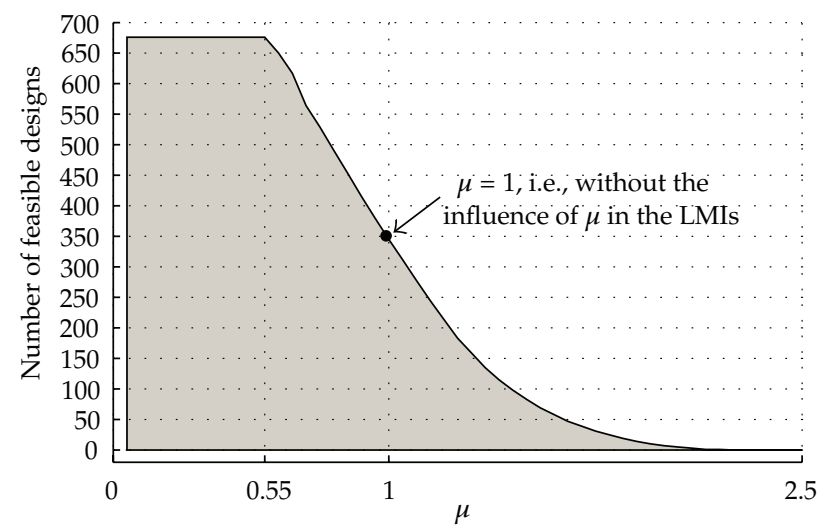

Figure 12: Parameters for which the controllers are found $(\mu \times$ Feasibility).

\section{Stability and Decay Rate}

Now, using Theorems 2.4 and 3.2 and considering $-10 \leq \zeta_{1} \leq 30$ and $0.4 \leq \zeta_{2} \leq 5.6$, the feasibility region with $\gamma=0.99$ is shown in Figure 13. Intentionally, the choice of $\gamma=0.99$ is due to the reason mentioned in Note 2.

Gradually increasing the value of the parameter $\gamma$, Theorem 3.2 can still find the controllers for stabilizing uncertain linear system, while designs without the parameter $\mu$ are no longer feasible. This is shown in Figure 14.

Considering the same uncertain linear system with $-20 \leq \zeta_{1} \leq 30$ and $-1.6 \leq \zeta_{2} \leq 2.4$, Figure 15 shows the totality of controllers that could be found for various values of $\gamma$ and $\mu$, comprising $\gamma \in[0,5]$ and $\mu \in[0.01,1.2]$. Analysing Figure 15, it can be observed that for a large value of $\gamma$, it is required a small value of $\mu$ for feasibility.

In this example, note that the values of $\mu$ are different for each design situation. After a one-dimensional search and visually considering Figures 12 and 15, these were the best values for the uncertain system. However, there is no theoretical evidence that these are the optimal values of $\mu$. 


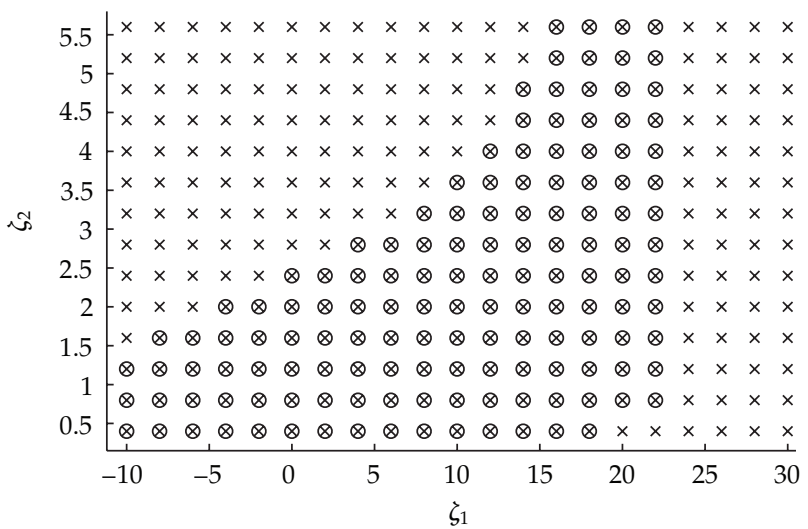

Figure 13: Controller design with Theorems $2.4(\circ)$ and $3.2(\mu=0.1, \times)$.

The next example illustrates a comparison of the stability criteria proposed in this paper with those proposed in [25] and in [31]. In [25], a CQLF is used to ensure the stability of the system with and without decay rate $\gamma>0$, and in [31], a PDLF is used for the same purposes.

\subsubsection{Example 3-Comparison between LMI-Based Techniques via State-Derivative Feedback Control}

Consider an uncertain linear system with the following polytope vertices:

$$
\begin{array}{ll}
A_{1}=\left[\begin{array}{cc}
21 & -98 \\
11 & 0
\end{array}\right], & B_{1}=\left[\begin{array}{c}
11 \\
0
\end{array}\right], \\
A_{2}=\left[\begin{array}{cc}
\zeta_{1} & -98 \\
11 & 32
\end{array}\right], & B_{2}=\left[\begin{array}{c}
\zeta_{2} \\
0
\end{array}\right] .
\end{array}
$$

\section{Stability}

Considering $-30 \leq \zeta_{1} \leq 102$ and $60 \leq \zeta_{2} \leq 111$, the region of feasibility considering only the stability of the system can be seen in Figure 16.

Note from Figure 16 that using only Theorem 2.3 from this work, which is more conservative than Theorem 3.1 of the same work, it has been possible to obtain less conservative conditions than those presented in [25] and in [31].

\section{Stability and Decay Rate}

Now, considering $-20 \leq \zeta_{1} \leq 40$ and $30 \leq \zeta_{2} \leq 55$, the feasibility region, considering stabilization with decay rate $\gamma=2$, is shown in Figure 17 .

Considering the restriction of decay rate $\gamma=2$, in this example it was used Theorem 3.2, knowing that Theorem 2.4 has no feasible answers for this value of $\gamma$. From Figure 17, note that the stability conditions proposed in this paper are less conservative than those presented in [25] and in [31]. Theorems 2.3 and 2.4 proposed in [31] have not been 


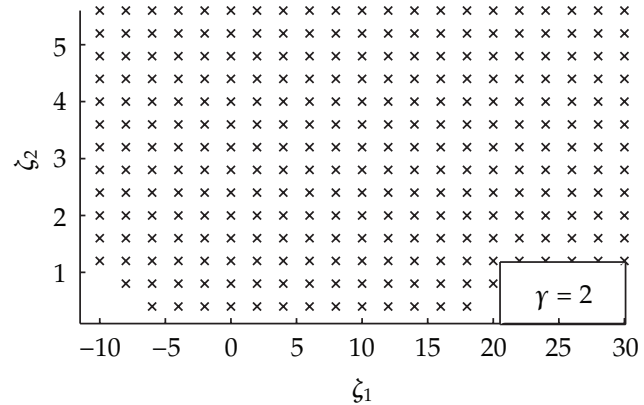

(a)

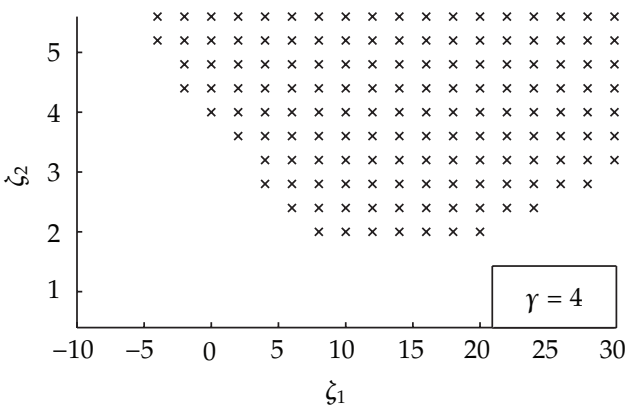

(c)

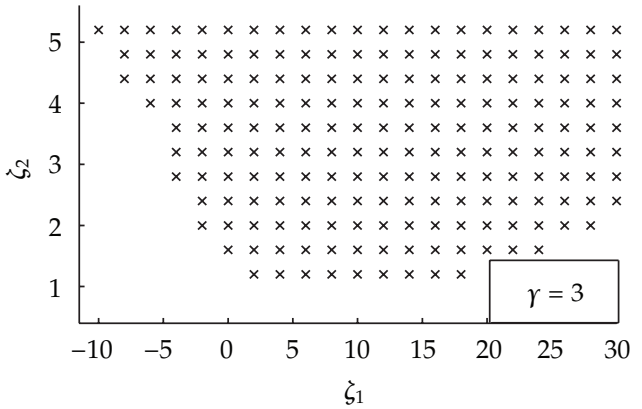

(b)

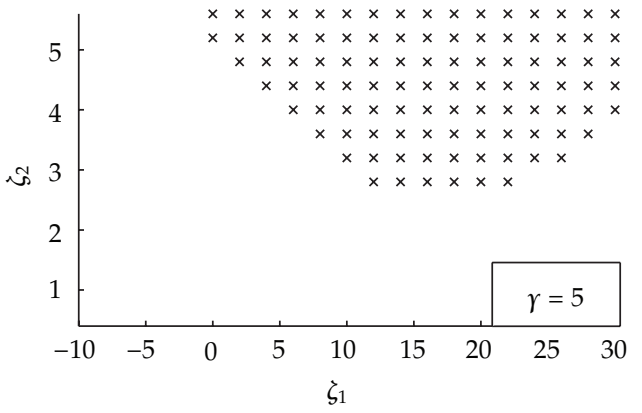

(d)

Figure 14: Controller design with Theorem $3.2(\mu=0.1, \times)$.

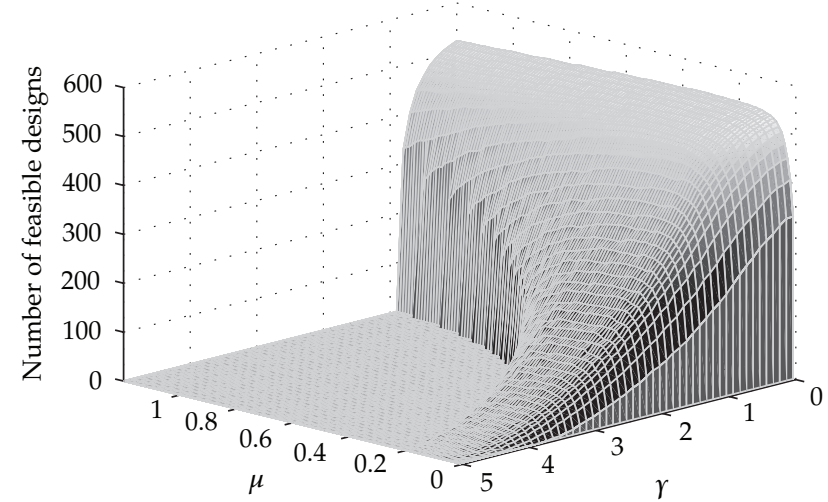

Figure 15: Parameters for which the controllers are found $(\mu \times \gamma \times$ feasibility).

used in both comparisons given that they are more conservative than Theorems 3.1 and 3.2 of the same work.

\section{Conclusions}

Sufficient conditions based on LMI directed for the state-derivative feedback controllers design, with and without decay rate restriction of uncertain linear systems using PDLFs, have been proposed. It is seen that the addition of the tuning parameter in LMI achieved better results, especially when considering the decay rate in the designs. The technique 


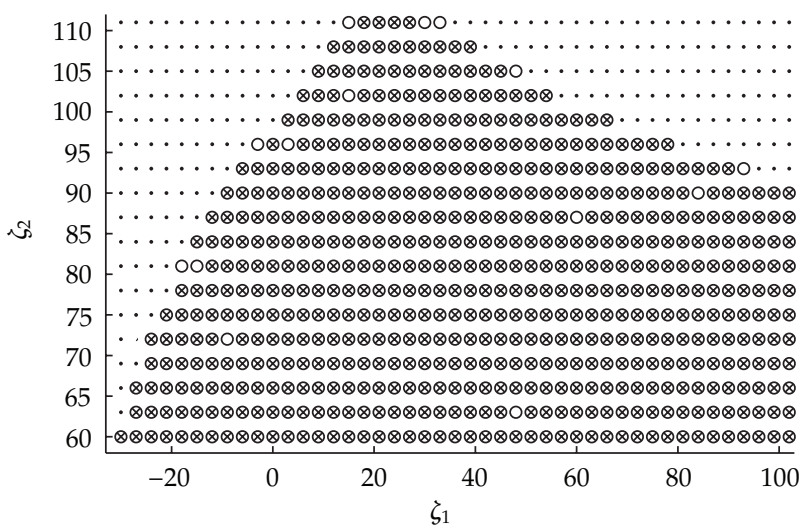

Figure 16: Controller design with da Silva et al. ([31, Theorem 3.1], $\varphi=200000, \times$ ), with Assunção et al. $([25$, Theorem 3$], \times$ and $\circ)$, and with Theorem 2.3 from this work $(x, \circ$ and $\cdot)$.

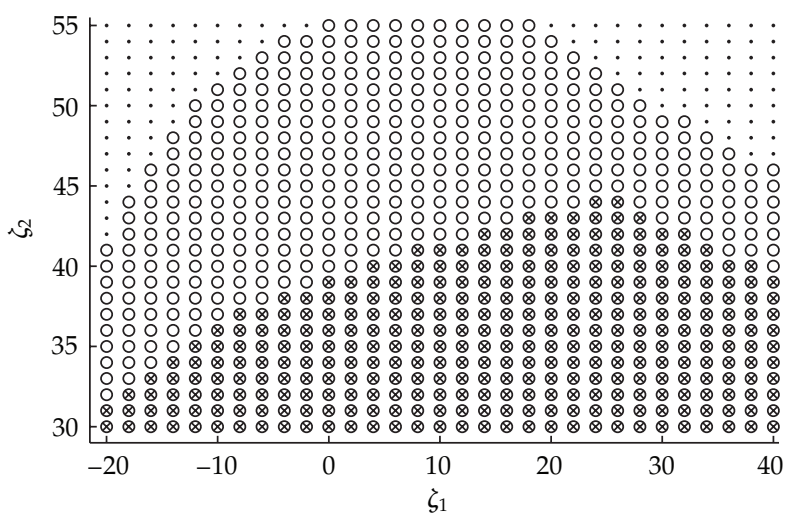

Figure 17: Controller design with Assunção et al. ([25, Theorem 4], $\times$ ), with da Silva et al. ([31, Theorem 3.2], $\varphi=0.2, \times$ and $\circ)$, and with Theorem 3.2 from this work $(\mu=0.1, \times, \circ$ and $\cdot)$.

is particularly interesting for applications in systems using accelerometers as sensors. The methodology addressed decouples the system matrices from the Lyapunov function matrices, thus improving the solution of problems. Comparisons between LMIs techniques using CQLFs and PDLFs functions were presented and discussed, and the results illustrate a less conservative search by controllers using the method proposed in this paper.

\section{Acknowledgments}

The authors would like to thank the Brazilian agencies CAPES, CNPq, and FAPESP which have supported this research.

\section{References}

[1] F. L. Lewis and V. L. Syrmos, "A geometric theory for derivative feedback," IEEE Transactions on Automatic Control, vol. 36, no. 9, pp. 1111-1116, 1991.

[2] T. H. S. Abdelaziz and M. Valášek, "Pole-placement for SISO linear systems by state-derivative feedback," vol. 151, no. 4, pp. 377-385. 
[3] Y. F. Duan, Y. Q. Ni, and J. M. Ko, "State-derivative feedback control of cable vibration using semiactive magnetorheological dampers," Computer-Aided Civil and Infrastructure Engineering, vol. 20, no. 6, pp. 431-449, 2005.

[4] S. K. Kwak, G. Washington, and R. K. Yedavalli, "Acceleration feedback-based active and passive vibration control of landing gear components," Journal of Aerospace Engineering, vol. 15, no. 1, pp. 1-9, 2002.

[5] E. Reithmeier and G. Leitmann, "Robust vibration control of dynamical systems based on the derivative of the state," Archive of Applied Mechanics, vol. 72, no. 11-12, pp. 856-864, 2003.

[6] E. R. P. da Silva, E. Assunção, M. C. M. Teixeira, and F. A. Faria, "Estabilização de sistemas fuzzy T-S incertos usando realimentação derivativa," SBA: Controle \& Automação Sociedade Brasileira de Automática, vol. 22, no. 3, pp. 273-283, 2011.

[7] T. H. S. Abdelaziz and M. Valášek, "State derivative feedback by LQR for linear timeinvariant systems," in the 16th IFAC World Congress, Czech Republic, 2005.

[8] S.-K. Kwak, G. Washington, and R. K. Yedavalli, "Acceleration-based vibration control of distributed parameter systems using the "reciprocal state-space framework"," Journal of Sound and Vibration, vol. 251, no. 3, pp. 543-557, 2002.

[9] M. R. Moreira, E. I. Mainardi Júnior, T. T. Esteves et al., "Stabilizability and disturbance rejection with state-derivative feedback," Mathematical Problems in Engineering, vol. 2010, Article ID 123751, 12 pages, 2010.

[10] R. Cardim, M. C. M. Teixeira, E. Assunção, and M. R. Covacic, “Design of state-derivative feedback controllers using a state feedback control design," in the 3rd IFAC Symposium on System, Structure and Control, vol. 1, pp. 135-141, Iguassu Falls, Brazil, 2007.

[11] J. M. Araújo, A. C. Castro, F. G. S. Silva, E. T. F. Santos, and C. E. T. Dórea, "Comparative study on state feedback and state-derivative feedback in linear time invariant systems," in the 3rd IFAC Symposium on System, Structure and Control, vol. 3, Iguassu Falls, Brazil, 2007.

[12] W. Michiels, T. Vyhlídal, H. Huijberts, and H. Nijmeijer, "Stabilizability and stability robustness of state derivative feedback controllers," SIAM Journal on Control and Optimization, vol. 47, no. 6, pp. 3100-3117, 2008.

[13] T. H. S. Abdelaziz and M. Valášek, "Direct algorithm for pole placement by state-derivative feedback for multi-input linear systems-nonsingular case," Kybernetika, vol. 41, no. 5, pp. 637-660, 2005.

[14] T. H. S. Abdelaziz and M. Valášek, "Eigenstructure assignment by proportional-plus-derivative feedback for second-order linear control systems," Kybernetika, vol. 41, no. 5, pp. 661-676, 2005.

[15] T. H. S. Abdelaziz, "Pole assignment by state-derivative feedback for single-input linear systems," Proceedings of the Institution of Mechanical Engineers, Part I, vol. 221, no. 7, pp. 991-1000, 2007.

[16] T. H. S. Abdelaziz, "Robust pole assignment for linear time-invariant systems using state-derivative feedback," Proceedings of the Institution of Mechanical Engineers. Part I, vol. 223, no. 2, pp. 187-199, 2009.

[17] T. H. S. Abdelaziz, "Optimal control using derivative feedback for linear systems," Proceedings of the Institution of Mechanical Engineers. Part I, vol. 224, no. 2, pp. 185-202, 2010.

[18] S. Boyd, L. El Ghaoui, E. Feron, and V. Balakrishnan, Linear Matrix Inequalities in System and Control Theory, vol. 15 of SIAM Studies in Applied Mathematics, SIAM, Philadelphia, Pa, USA, 1994.

[19] P. Gahinet, A. Nemirovski, A. J. Laub, and M. Chilali, LMI control toolbox - For use with MATLAB, The Math Works Inc., 1995.

[20] R. Cardim, M. C. M. Teixeira, E. Assunção, and F. A. Faria, “Control designs for linear systems using state-derivative feedback," in Systems, Structure and Control, pp. 1-28, InTech, Vienna, Austria, 2008.

[21] R. Cardim, M. C. M. Teixeira, F. A. Faria, and E. Assunção, "LMI-based digital redesign of linear time-invariant systems with state-derivative feedback," in IEEE International Conference on Control Applications (CCA '09), pp. 745-749, July 2009.

[22] Y. Jing, C. Shen, G. M. Dimirovski, and N. Jiang, "Delay-dependent state-derivative feedback with an $\alpha$-stability constraint for time delay systems," in American Control Conference (ACC '09), pp. 1844-1848, June 2009.

[23] F. A. Faria, E. Assunção, M. C. M. Teixeira, R. Cardim, and N. A. P. da Silva, "Robust state-derivative pole placement LMI-based designs for linear systems," International Journal of Control, vol. 82, no. 1, pp. 1-12, 2009.

[24] F. A. Faria, E. Assunção, M. C. M. Teixeira, and R. Cardim, "Robust state-derivative feedback LMIbased designs for linear descriptor systems," Mathematical Problems in Engineering, vol. 2010, Article ID 927362, 15 pages, 2010. 
[25] E. Assunção, M. C. M. Teixeira, F. A. Faria, N. A. P. Da Silva, and R. Cardim, "Robust state-derivative feedback LMI-based designs for multivariable linear systems," International Journal of Control, vol. 80, no. 8, pp. 1260-1270, 2007.

[26] R. C. L. F. Oliveira and P. L. D. Peres, "LMI conditions for robust stability analysis based on polynomially parameter-dependent Lyapunov functions," Systems \& Control Letters, vol. 55, no. 1, pp. 52-61, 2006.

[27] M. C. de Oliveira and R. E. Skelton, "Stability tests for constrained linear systems," in Perspectives in Robust Control, vol. 268 of Lecture Notes in Control and Inform. Sci., pp. 241-257, Springer, London, UK, 2001.

[28] M. C. de Oliveira and J. C. Geromel, "A class of robust stability conditions where linear parameter dependence of the Lyapunov function is a necessary condition for arbitrary parameter dependence," Systems \& Control Letters, vol. 54, no. 11, pp. 1131-1134, 2005.

[29] S.-W. Kau, Y.-S. Liu, L. Hong, C.-H. Lee, C.-H. Fang, and L. Lee, “A new LMI condition for robust stability of discrete-time uncertain systems," Systems $\mathcal{E}$ Control Letters, vol. 54, no. 12, pp. 1195-1203, 2005.

[30] J. C. Geromel and R. H. Korogui, "Analysis and synthesis of robust control systems using linear parameter dependent Lyapunov functions," IEEE Transactions on Automatic Control, vol. 51, no. 12, pp. 1984-1989, 2006.

[31] E. R. P. Da Silva, E. Assuncao, M. C. M. Teixeira, F. A. Faria, and L. F. S. Buzachero, "Parameterdependent Lyapunov functions for state-derivative feedback control in polytopic linear systems," International Journal of Control, vol. 84, no. 8, pp. 1377-1386, 2011.

[32] R. E. Skelton, T. Iwasaki, and K. M. Grigoriadis, A Unified Algebraic Approach to Linear Control Design, The Taylor \& Francis Systems and Control Book Series, Taylor \& Francis, London, UK, 1998.

[33] G. Pipeleers, B. Demeulenaere, J. Swevers, and L. Vandenberghe, “Extended LMI characterizations for stability and performance of linear systems," Systems $\mathcal{E}$ Control Letters, vol. 58, no. 7, pp. 510-518, 2009.

[34] L. A. Mozelli, R. M. Palhares, and E. M. A. M. Mendes, “Equivalent techniques, extra comparisons and less conservative control design for Takagi-Sugeno (TS) fuzzy systems," IET Control Theory $\mathcal{E}$ Applications, vol. 4, no. 12, pp. 2813-2822, 2010.

[35] L. A. Mozelli, R. M. Palhares, and G. S. C. Avellar, "A systematic approach to improve multiple Lyapunov function stability and stabilization conditions for fuzzy systems," Information Sciences, vol. 179, no. 8, pp. 1149-1162, 2009. 


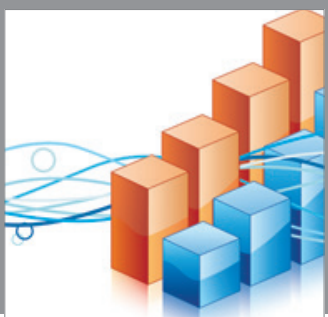

Advances in

Operations Research

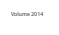

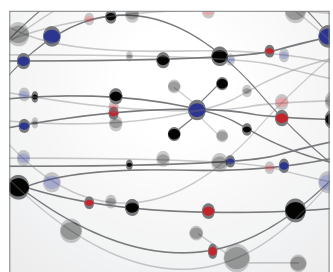

\section{The Scientific} World Journal
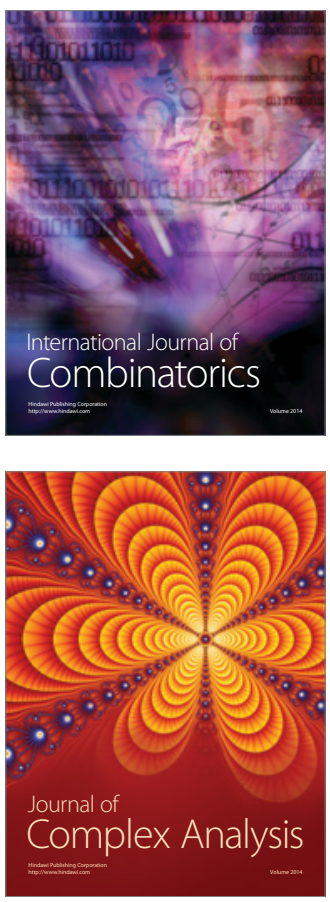

International Journal of

Mathematics and

Mathematical

Sciences
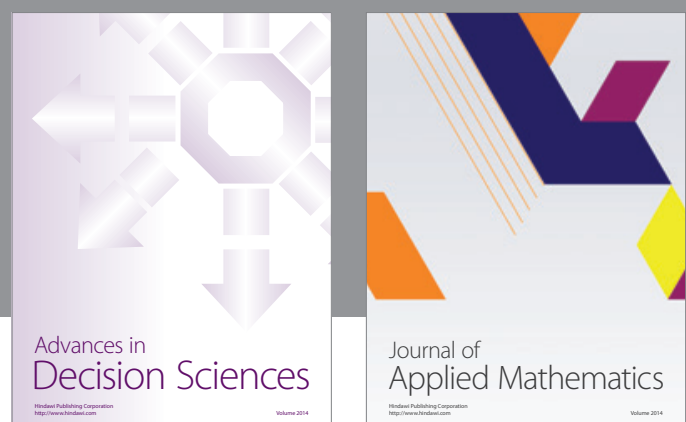

Journal of

Applied Mathematics
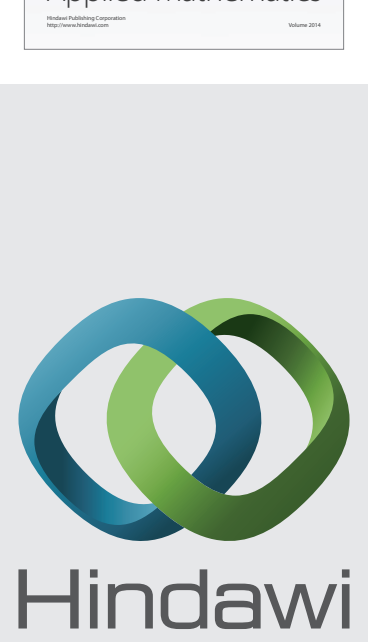

Submit your manuscripts at http://www.hindawi.com
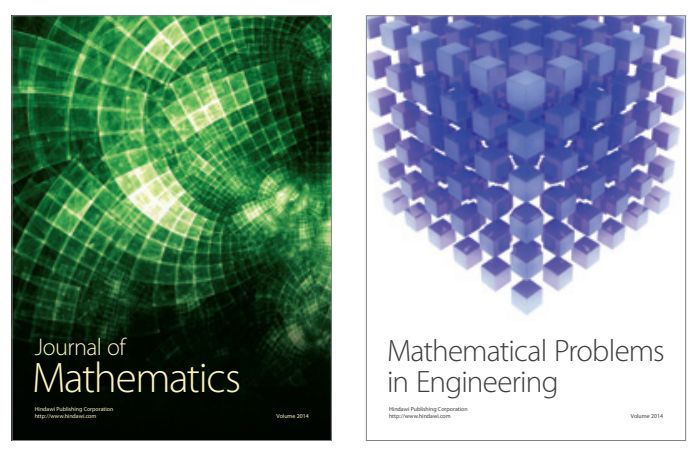

Mathematical Problems in Engineering
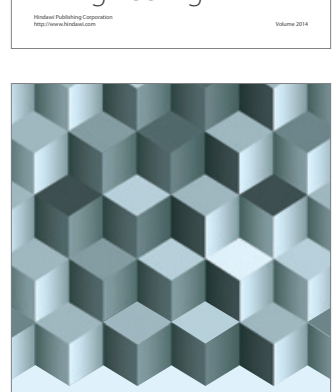

Journal of

Function Spaces
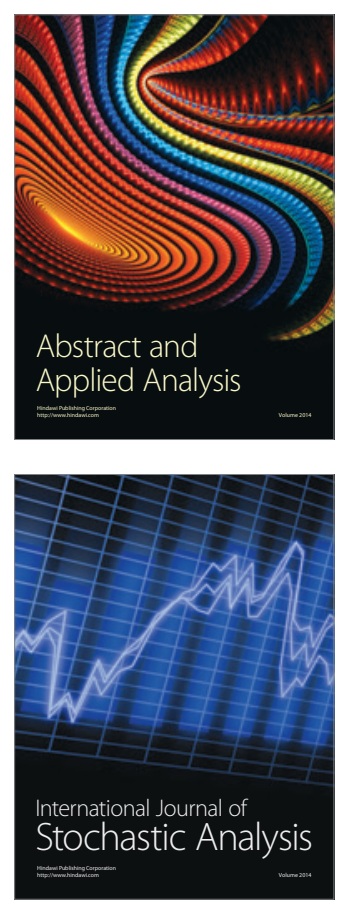

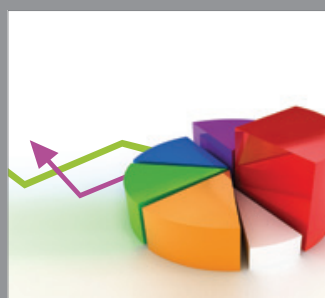

ournal of

Probability and Statistics

Promensencen
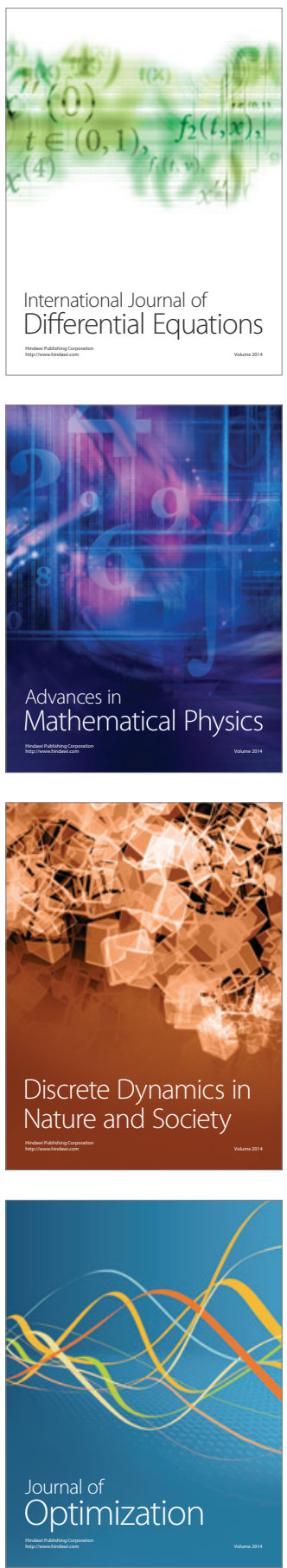